PROCEEDINGS OF THE

AMERICAN MATHEMATICAL SOCIETY

Volume 140, Number 6, June 2012, Pages 2033-2042

S 0002-9939(2011)11073-6

Article electronically published on October 13, 2011

\title{
$A$-HYPERGEOMETRIC SYSTEMS THAT COME FROM GEOMETRY
}

\author{
ALAN ADOLPHSON AND STEVEN SPERBER
}

(Communicated by Lev Borisov)

\begin{abstract}
In recent work, Beukers characterized $A$-hypergeometric systems having a full set of algebraic solutions. He accomplished this by (1) determining which $A$-hypergeometric systems have a full set of polynomial solutions modulo $p$ for almost all primes $p$ and (2) showing that these systems come from geometry. He then applied a fundamental theorem of N. Katz, which says that such systems have a full set of algebraic solutions. In this paper we establish some connections between nonresonant $A$-hypergeometric systems and de Rham-type complexes, which leads to a determination of which $A$ hypergeometric systems come from geometry. We do not use the fact that the system is irreducible or find integral formulas for its solutions.
\end{abstract}

\section{INTRODUCTION}

Let $A=\left\{a^{(1)}, \ldots, a^{(N)}\right\} \subseteq \mathbb{Z}^{n}$ with $a^{(j)}=\left(a_{1}^{(j)}, \ldots, a_{n}^{(j)}\right)$. We shall assume throughout this paper that these lattice points generate $\mathbb{Z}^{n}$ as an abelian group. Let $L$ be the corresponding lattice of relations,

$$
L=\left\{\left(l_{1}, \ldots, l_{N}\right) \in \mathbb{Z}^{N} \mid \sum_{j=1}^{N} l_{j} a^{(j)}=0\right\},
$$

and let $\alpha=\left(\alpha_{1}, \ldots, \alpha_{n}\right) \in \mathbb{C}^{n}$. The A-hypergeometric system is the system of partial differential equations in the variables $\lambda_{1}, \ldots, \lambda_{N}$ consisting of the operators (we write $\partial_{j}$ for $\partial / \partial \lambda_{j}$ )

$$
\square_{l}=\prod_{l_{j}>0} \partial_{j}^{l_{j}}-\prod_{l_{j}<0} \partial_{j}^{-l_{j}}
$$

for all $l \in L$ and the operators

$$
Z_{i, \alpha}=\sum_{j=1}^{N} a_{i}^{(j)} \lambda_{j} \partial_{j}-\alpha_{i}
$$

Received by the editors December 9, 2010 and, in revised form, January 24, 2011 and February $9,2011$.

2010 Mathematics Subject Classification. Primary 33C70, 14F40; Secondary 52B20.

Key words and phrases. A-hypergeometric system, de Rham cohomology.

(C)2011 American Mathematical Society 
for $i=1, \ldots, n$. We denote by $\mathcal{D}=\mathbb{C}\left\langle\lambda_{1}, \ldots, \lambda_{N}, \partial_{1}, \ldots, \partial_{N}\right\rangle$ the ring of differential operators in the $\lambda_{j}$. The associated hypergeometric $\mathcal{D}$-module is

$$
\mathcal{M}_{\alpha}=\mathcal{D} /\left(\sum_{l \in L} \mathcal{D} \square_{l}+\sum_{i=1}^{n} \mathcal{D} Z_{i, \alpha}\right) .
$$

Let $\mathbb{C}[\lambda]=\mathbb{C}\left[\lambda_{1}, \ldots, \lambda_{N}\right]$ be the polynomial ring in $N$ variables and let $X$ be a smooth variety over $\mathbb{C}[\lambda]$. Let $G$ be a finite group acting on $X / \mathbb{C}[\lambda]$. Then $G$ acts on the relative de Rham cohomology groups $H_{\mathrm{DR}}^{i}(X / \mathbb{C}[\lambda])$. For an irreducible representation $\chi$ of $G$, let $H_{\mathrm{DR}}^{i}(X / \mathbb{C}[\lambda])^{\chi}$ denote the $\chi$-isotypic component of $H_{\mathrm{DR}}^{i}(X / \mathbb{C}[\lambda])$; i.e., $H_{\mathrm{DR}}^{i}(X / \mathbb{C}[\lambda])^{\chi}$ is the sum of all $G$-submodules of $H_{\mathrm{DR}}^{i}(X / \mathbb{C}[\lambda])$ that are isomorphic to $\chi$. The $H_{\mathrm{DR}}^{i}(X / \mathbb{C}[\lambda])^{\chi}$ are $\mathcal{D}$-modules via the Gauss-Manin connection. We say that a $\mathcal{D}$-module $\mathcal{M}$ comes from geometry if it is isomorphic to $H_{\mathrm{DR}}^{i}(X / \mathbb{C}[\lambda])^{\chi}$ for some $(X / \mathbb{C}[\lambda], G, \chi)$.

The main idea of this paper is to show that the $\mathcal{M}_{\alpha}$ are isomorphic as $\mathcal{D}$ modules to certain cohomology groups that arise in algebraic geometry. Let $R^{\prime}=$ $\mathbb{C}[\lambda]\left[x_{1}^{ \pm 1}, \ldots, x_{n}^{ \pm 1}\right]$, the coordinate ring of the $n$-torus $\mathbb{T}^{n}$ over $\mathbb{C}[\lambda]$. Put

$$
f=\sum_{j=1}^{N} \lambda_{j} x^{a^{(j)}} \in R^{\prime}
$$

and let $\Omega_{R^{\prime} / \mathbb{C}[\lambda]}^{k}$ denote the module of relative $k$-forms. We use the set

$$
\left\{\frac{d x_{i_{1}}}{x_{i_{1}}} \wedge \cdots \wedge \frac{d x_{i_{k}}}{x_{i_{k}}} \mid 1 \leq i_{1}<\cdots<i_{k} \leq n\right\}
$$

as a basis for $\Omega_{R^{\prime} / \mathbb{C}[\lambda]}^{k}$ as an $R^{\prime}$-module. The map $\nabla_{\alpha}: \Omega_{R^{\prime} / \mathbb{C}[\lambda]}^{k} \rightarrow \Omega_{R^{\prime} / \mathbb{C}[\lambda]}^{k+1}$ given by

$$
\nabla_{\alpha}(\omega)=d \omega+\sum_{k=1}^{n} \alpha_{i} \frac{d x_{i}}{x_{i}} \wedge \omega+d f \wedge \omega
$$

defines a complex $\left(\Omega_{R^{\prime} / \mathbb{C}[\lambda]}^{\bullet}, \nabla_{\alpha}\right)$. In terms of the above basis, we have for $\xi \in R^{\prime}$

$$
\nabla_{\alpha}\left(\xi \frac{d x_{i_{1}}}{x_{i_{1}}} \wedge \cdots \wedge \frac{d x_{i_{k}}}{x_{i_{k}}}\right)=\left(\sum_{i=1}^{n} D_{i, \alpha}(\xi) \frac{d x_{i}}{x_{i}}\right) \wedge \frac{d x_{i_{1}}}{x_{i_{1}}} \wedge \cdots \wedge \frac{d x_{i_{k}}}{x_{i_{k}}},
$$

where

$$
D_{i, \alpha}=x_{i} \frac{\partial}{\partial x_{i}}+\alpha_{i}+x_{i} \frac{\partial f}{\partial x_{i}}
$$

Formally,

$$
\nabla_{\alpha}=\frac{1}{x^{\alpha} \exp f} \circ d \circ x^{\alpha} \exp f
$$

so for any derivation $\partial \in \operatorname{Der}_{\mathbb{C}}(\mathbb{C}[\lambda])$ the operator

$$
D_{\partial}=\frac{1}{x^{\alpha} \exp f} \circ \partial \circ x^{\alpha} \exp f=\partial+f^{\partial}
$$

on the $\Omega_{R^{\prime} / \mathbb{C}[\lambda]}^{k}$ commutes with $\nabla_{\alpha}$ (where $f^{\partial}$ denotes the polynomial obtained from $f$ by applying $\partial$ to its coefficients). This defines an action of $\operatorname{Der}_{\mathbb{C}}(\mathbb{C}[\lambda])$ on $\left(\Omega_{R^{\prime} / \mathbb{C}[\lambda]}^{\bullet}, \nabla_{\alpha}\right)$. In particular, $\partial_{j}$ acts as $D_{j}=\partial_{j}+x^{a^{(j)}}$. This action extends to an action of $\mathcal{D}$ on $\left(\Omega_{R^{\prime} / \mathbb{C}[\lambda]}^{\bullet}, \nabla_{\alpha}\right)$, which makes the $H^{i}\left(\Omega_{R^{\prime} / \mathbb{C}[\lambda]}^{\bullet}, \nabla_{\alpha}\right)$ into $\mathcal{D}$-modules. 
Let $C(A) \subseteq \mathbb{R}^{n}$ be the real cone generated by $A$ and let $\ell_{1}, \ldots, \ell_{s} \in \mathbb{Z}\left[u_{1}, \ldots, u_{n}\right]$ be homogeneous linear forms defining the codimension-one faces of $C(A)$, normalized so that the coefficients of each $\ell_{i}$ are relatively prime and so that $\ell_{i} \geq 0$ on $C(A)$ for each $i$. We say that $\alpha$ is nonresonant for $A$ if $\ell_{i}(\alpha) \notin \mathbb{Z}$ for all $i$. Note that $\alpha$ is nonresonant for $A$ if and only if $\alpha+u$ is nonresonant for $A$ for all $u \in \mathbb{Z}^{n}$. (If $\alpha \in \mathbb{R}^{n}$, this is equivalent to saying that no proper face of $C(A)$ contains a point of $\alpha+\mathbb{Z}^{n}$.)

Theorem 1.4. If $\alpha$ is nonresonant for $A$, then $\mathcal{M}_{\alpha} \cong H^{n}\left(\Omega_{R^{\prime} / \mathbb{C}[\lambda]}, \nabla_{\alpha}\right)$ as $\mathcal{D}$ modules.

Remark 1. One can show that the complex $\left(\Omega_{R^{\prime} / \mathbb{C}[\lambda]}^{\bullet}, \nabla_{\alpha}\right)$ is isomorphic to the Euler-Koszul complex on the localization denoted $S_{A}\left[\partial_{A}^{-1}\right]$ in Schulze-Walther [14]. Theorem 1.4 then follows from results in [14, Sections 2 and 3]. We believe the approach we follow here to be more convenient for explaining the relationship with the relative de Rham cohomology of algebraic varieties. By [14, Sections 2 and 3], this Euler-Koszul complex is in fact a resolution of $\mathcal{M}_{\alpha}$ for $\alpha$ nonresonant, so $\left(\Omega_{R^{\prime} / \mathbb{C}[\lambda]}^{\bullet}, \nabla_{\alpha}\right)$ is also a resolution of $\mathcal{M}_{\alpha}$.

Remark 2. It is straightforward to check that for $u \in \mathbb{Z}^{n}$ multiplication by $x^{u}$ defines an isomorphism of complexes of $\mathcal{D}$-modules

$$
x^{u}:\left(\Omega_{R^{\prime} / \mathbb{C}[\lambda]}^{\bullet}, \nabla_{\alpha+u}\right) \rightarrow\left(\Omega_{R^{\prime} / \mathbb{C}[\lambda]}^{\bullet}, \nabla_{\alpha}\right)
$$

(its inverse is multiplication by $x^{-u}$ ). Thus when $\alpha$ is nonresonant the $\mathcal{M}_{\alpha+u}$ for $u \in \mathbb{Z}^{n}$ are all isomorphic as $\mathcal{D}$-modules.

Consider the special case $f=x_{n} g$, where $g \in \mathbb{C}[\lambda]\left[x_{1}^{ \pm 1}, \ldots, x_{n-1}^{ \pm 1}\right]$. Let $\mathbb{T}^{n-1}$ be the $(n-1)$-torus over $\mathbb{C}[\lambda]$ with coordinates $x_{1}, \ldots, x_{n-1}$. Let $U \subseteq \mathbb{T}^{n-1}$ be the open set where $g$ is nonvanishing and let $\Omega_{U / \mathbb{C}[\lambda]}^{k}$ be the module of relative $k$-forms over the ring of regular functions on $U$. The map $\widetilde{\nabla}_{\alpha}: \Omega_{U / \mathbb{C}[\lambda]}^{k} \rightarrow \Omega_{U / \mathbb{C}[\lambda]}^{k+1}$ defined by

$$
\widetilde{\nabla}_{\alpha}(\omega)=d \omega+\sum_{i=1}^{n-1} \alpha_{i} \frac{d x_{i}}{x_{i}} \wedge \omega-\alpha_{n} \frac{d g}{g} \wedge \omega
$$

defines a complex $\left(\Omega_{U / \mathbb{C}[\lambda]}^{\bullet}, \widetilde{\nabla}_{\alpha}\right)$. Formally we have

$$
\widetilde{\nabla}_{\alpha}=\frac{g^{\alpha_{n}}}{x_{1}^{\alpha_{1}} \cdots x_{n}^{\alpha_{n}}} \circ d \circ \frac{x_{1}^{\alpha_{1}} \cdots x_{n}^{\alpha_{n}}}{g^{\alpha_{n}}},
$$

so if we define for $\partial \in \operatorname{Der}_{\mathbb{C}}(\mathbb{C}[\lambda])$

$$
\widetilde{D}_{\partial}=\frac{g^{\alpha_{n}}}{x_{1}^{\alpha_{1}} \cdots x_{n}^{\alpha_{n}}} \circ \partial \circ \frac{x_{1}^{\alpha_{1}} \cdots x_{n}^{\alpha_{n}}}{g^{\alpha_{n}}}=\partial-\alpha_{n} \frac{g^{\partial}}{g},
$$

we get an action of $\operatorname{Der}_{\mathbb{C}}(\mathbb{C}[\lambda])$ on this complex. The action extends to an action of $\mathcal{D}$, making $\left(\Omega_{U / \mathbb{C}[\lambda]}^{\bullet}, \widetilde{\nabla}_{\alpha}\right)$ into a complex of $\mathcal{D}$-modules. Note that for $u \in \mathbb{Z}^{n}$, multiplication by $x_{1}^{u_{1}} \cdots x_{n-1}^{u_{n-1}} / g^{u_{n}}$ defines an isomorphism of complexes of $\mathcal{D}$-modules

$$
\frac{x_{1}^{u_{1}} \cdots x_{n-1}^{u_{n-1}}}{g^{u_{n}}}:\left(\Omega_{U / \mathbb{C}[\lambda]}^{\bullet}, \widetilde{\nabla}_{\alpha+u}\right) \rightarrow\left(\Omega_{U / \mathbb{C}[\lambda]}^{\bullet}, \widetilde{\nabla}_{\alpha}\right) .
$$


Theorem 1.5. Suppose $f=x_{n} g\left(x_{1}, \ldots, x_{n-1}\right)$ and $\alpha$ is nonresonant for $A$. For all $i$ there are $\mathcal{D}$-module isomorphisms

$$
H^{i}\left(\Omega_{R^{\prime} / \mathbb{C}[\lambda]}^{\bullet}, \nabla_{\alpha}\right) \cong H^{i-1}\left(\Omega_{U / \mathbb{C}[\lambda]}^{\bullet}, \widetilde{\nabla}_{\alpha}\right) .
$$

Remark 3. In view of Remark 1 , we have $H^{i}\left(\Omega_{R^{\prime} / \mathbb{C}[\lambda]}, \nabla_{\alpha}\right)=0$ for $i \neq n$, so both sides of the isomorphism of Theorem 1.5 vanish for $i \neq n$.

When $\alpha \in \mathbb{Q}^{n}$, it is well known that the $H^{i}\left(\Omega_{U / \mathbb{C}[\lambda]}^{\bullet}, \widetilde{\nabla}_{\alpha}\right)$ come from geometry. (We sketch the proof of this fact in Section 4.) From Theorems 1.4 and 1.5, we then get the following result.

Corollary 1.6. Suppose $f=x_{n} g\left(x_{1}, \ldots, x_{n-1}\right)$ and $\alpha$ is nonresonant for $A$. There is an isomorphism of $\mathcal{D}$-modules $\mathcal{M}_{\alpha} \cong H^{n-1}\left(\Omega_{U / \mathbb{C}[\lambda]}^{\bullet}, \widetilde{\nabla}_{\alpha}\right)$. If in addition $\alpha \in \mathbb{Q}^{n}$, then $\mathcal{M}_{\alpha}$ comes from geometry.

The proofs of Theorems 1.4 and 1.5 are based on ideas from [1] and [3]. Those papers in turn are related to earlier work of Dwork, Dwork-Loeser, and N. Katz. We refer the reader to the introductions of [1] and 3] for more details on the connections with that earlier work.

\section{Proof of Theorem 1.4}

It is straightforward to check that the $Z_{i, \alpha}$ commute with one another and that

$$
\square_{l} \circ Z_{i, \alpha}=Z_{i, \beta} \circ \square_{l},
$$

where $\beta=\alpha+\sum_{l_{j}>0} l_{j} a^{(j)} \quad\left(=\alpha-\sum_{l_{j}<0} l_{j} a^{(j)}\right.$ since $\left.l \in L\right)$. It follows that right multiplication by $Z_{i, \alpha}$ maps the left ideal $\sum_{l \in L} \mathcal{D} \square_{l}$ into itself. If we put $\mathcal{P}=\mathcal{D} / \sum_{l \in L} \mathcal{D} \square_{l}$, then right multiplication by the $Z_{i, \alpha}$ is a family of commuting endomorphisms of $\mathcal{P}$ as a left $\mathcal{D}$-module. Let $\mathcal{C} \bullet$ be the cohomological Koszul complex on $\mathcal{P}$ defined by the $Z_{i, \alpha}$. Concretely,

$$
\mathcal{C}^{k}=\bigoplus_{1 \leq i_{1}<\cdots<i_{k} \leq n} \mathcal{P} e_{i_{1}} \wedge \cdots \wedge e_{i_{k}},
$$

where the $e_{i}$ are formal symbols satisfying $e_{i} \wedge e_{j}=-e_{j} \wedge e_{i}$ and the boundary operator $\delta_{\alpha}: \mathcal{C}^{k} \rightarrow \mathcal{C}^{k+1}$ is defined by additivity and the formula (for $\sigma \in \mathcal{P}$ )

$$
\delta_{\alpha}\left(\sigma e_{i_{1}} \wedge \cdots \wedge e_{i_{k}}\right)=\sum_{i=1}^{n} \sigma Z_{i, \alpha} e_{i} \wedge e_{i_{1}} \wedge \cdots \wedge e_{i_{k}} .
$$

One obtains a complex of left $\mathcal{D}$-modules $\left(\mathcal{C}^{\bullet}, \delta_{\alpha}\right)$ for which

$$
H^{n}\left(\mathcal{C}^{\bullet}, \delta_{\alpha}\right)=\mathcal{M}_{\alpha}
$$

Let $R=\mathbb{C}[\lambda]\left[x^{a^{(1)}}, \ldots, x^{a^{(N)}}\right]$, a subring of $R^{\prime}$ which is also a $\mathcal{D}$-submodule of $R^{\prime}$, and let

$$
\Omega_{R}^{k}\langle\log \rangle=\left\{\sum_{1 \leq i_{1}<\cdots<i_{k} \leq n} \xi_{i_{1} \cdots i_{k}} \frac{d x_{i_{1}}}{x_{i_{1}}} \wedge \cdots \wedge \frac{d x_{i_{k}}}{x_{i_{k}}} \mid \xi_{i_{1} \cdots i_{k}} \in R\right\} \subseteq \Omega_{R^{\prime} / \mathbb{C}[\lambda]}^{k} .
$$

By (1.2) and (1.3), one has $\nabla_{\alpha}\left(\Omega_{R}^{k}\langle\log \rangle\right) \subseteq \Omega_{R}^{k+1}\langle\log \rangle$, so this defines a subcomplex $\left(\Omega_{R}^{\bullet}\langle\log \rangle, \nabla_{\alpha}\right)$ of $\left(\Omega_{R^{\prime} / \mathbb{C}[\lambda]}^{\bullet}, \nabla_{\alpha}\right)$.

The $\mathbb{C}[\lambda]$-module homomorphism $\phi: \mathcal{P} \rightarrow R$ defined by

$$
\phi\left(\partial_{1}^{b_{1}} \cdots \partial_{N}^{b_{N}}\right)=x^{\sum_{j=1}^{N} b_{j} a^{(j)}}
$$


is an isomorphism of $\mathcal{D}$-modules by [1, Theorem 4.4]. It extends to a map $\phi: \mathcal{C}^{k} \rightarrow$ $\Omega_{R}^{k}\langle\log \rangle$ by additivity and the formula

$$
\phi\left(\sigma e_{i_{1}} \wedge \cdots \wedge e_{i_{k}}\right)=\phi(\sigma) \frac{d x_{i_{1}}}{x_{i_{1}}} \wedge \cdots \wedge \frac{d x_{i_{k}}}{x_{i_{k}}} .
$$

By [2, Corollary 2.4], this is an isomorphism of complexes of $\mathcal{D}$-modules:

$$
\phi:\left(\mathcal{C}^{\bullet}, \delta_{\alpha}\right) \stackrel{\cong}{\rightarrow}\left(\Omega_{R}^{\bullet}\langle\log \rangle, \nabla_{\alpha}\right) .
$$

By (2.1) and (2.2), Theorem 1.4 is a consequence of the following result.

Proposition 2.3. If $\alpha$ is nonresonant for $A$, then the inclusion map

$$
\left(\Omega_{R}^{\bullet}\langle\log \rangle, \nabla_{\alpha}\right) \hookrightarrow\left(\Omega_{R^{\prime} / \mathbb{C}[\lambda]}^{\bullet}, \nabla_{\alpha}\right)
$$

is a quasi-isomorphism of complexes of $\mathcal{D}$-modules.

We state and prove a generalization of Proposition 2.3. Let $U \subseteq \mathbb{Z}^{n}$ be a nonempty subset satisfying the following condition:

$$
\text { if } u \in U \text {, then } u+a^{(j)} \in U \text { for } j=1, \ldots, N \text {. }
$$

If we denote by $M_{U}$ the free $\mathbb{C}[\lambda]$-module with basis $\left\{x^{u} \mid u \in U\right\}$, then (2.4) implies that $M_{U}$ is both an $R$-submodule and a $\mathcal{D}$-submodule of $R^{\prime}$. Note that $R^{\prime}=M_{\mathbb{Z}^{n}}$ and that $R=M_{U_{0}}$, where $U_{0}=\left\{\sum_{j=1}^{N} c_{j} a^{(j)} \mid c_{j} \in \mathbb{Z}_{\geq 0}\right\}$. Let $\Omega_{M_{U}}^{k}\langle\log \rangle \subseteq \Omega_{R^{\prime} / \mathbb{C}[\lambda]}^{k}$ be the subset

$$
\Omega_{M_{U}}^{k}\langle\log \rangle=\left\{\sum_{1 \leq i_{1}<\cdots<i_{k} \leq n} \xi_{i_{1} \cdots i_{k}} \frac{d x_{i_{1}}}{x_{i_{1}}} \wedge \cdots \wedge \frac{d x_{i_{k}}}{x_{i_{k}}} \mid \xi_{i_{1} \cdots i_{k}} \in M_{U}\right\} .
$$

By (1.2) and (1.3), we have $\nabla_{\alpha}\left(\Omega_{M_{U}}^{k}\langle\log \rangle\right) \subseteq \Omega_{M_{U}}^{k+1}\langle\log \rangle$, so we get a subcomplex $\left(\Omega_{M_{U}}^{\bullet}\langle\log \rangle, \nabla_{\alpha}\right)$ of $\left(\Omega_{R^{\prime} / \mathbb{C}[\lambda]}^{\bullet}, \nabla_{\alpha}\right)$. Proposition 2.3 is the special case $U=U_{0}$ of the following more general result.

Proposition 2.5. If $\alpha$ is nonresonant for $A$ and $U$ satisfies (2.4), then the inclusion

$$
\left(\Omega_{M_{U}}^{\bullet}\langle\log \rangle, \nabla_{\alpha}\right) \hookrightarrow\left(\Omega_{R^{\prime} / \mathbb{C}[\lambda]}^{\bullet}, \nabla_{\alpha}\right)
$$

is a quasi-isomorphism of complexes of $\mathcal{D}$-modules.

As in Section 1 , let $\ell_{1}, \ldots, \ell_{s} \in \mathbb{Z}\left[u_{1}, \ldots, u_{n}\right]$ be the normalized homogeneous linear forms defining the codimension-one faces of $C(A)$ and put $S=\{1, \ldots, s\}$. For $v=\left(v_{1}, \ldots, v_{s}\right) \in \mathbb{Z}^{s}$ and a subset $T \subseteq S$, put

$$
W(v, T)=\left\{u \in \mathbb{Z}^{n} \mid \ell_{i}(u) \geq v_{i} \text { for all } i \in T\right\} .
$$

Since $\ell_{i}\left(a^{(j)}\right) \geq 0$ for all $i, j$, the set $W(v, T)$ satisfies (2.4). By [1, Lemma 3.12], there exists $v$ such that $W(v, S) \subseteq U_{0}$. It follows that if $U$ satisfies (2.4), then there exists $v$ such that $W(v, S) \subseteq U$.

Lemma 2.6. If $\alpha$ is nonresonant for $A, U$ satisfies (2.4), and $W(v, T) \subseteq U$, then the inclusion

$$
\left(\Omega_{M_{W(v, T)}^{\bullet}}\langle\log \rangle, \nabla_{\alpha}\right) \hookrightarrow\left(\Omega_{M_{U}}^{\bullet}\langle\log \rangle, \nabla_{\alpha}\right)
$$

is a quasi-isomorphism of complexes of $\mathcal{D}$-modules. 
Since $W(v, T) \subseteq \mathbb{Z}^{n}$ for all $v, T$, Lemma 2.6 implies that the inclusion

$$
\left(\Omega_{M_{W(v, T)}}^{\bullet}\langle\log \rangle, \nabla_{\alpha}\right) \hookrightarrow\left(\Omega_{R^{\prime} / \mathbb{C}[\lambda]}^{\bullet}, \nabla_{\alpha}\right)
$$

is a quasi-isomorphism for all $v, T$. The quasi-isomorphisms (2.7) and (2.8) imply Proposition 2.5.

Proof of Lemma 2.6. For $T^{\prime} \subseteq T$, let

$$
U\left(v, T^{\prime}\right)=\left\{u \in U \mid \ell_{i}(u) \geq v_{i} \text { for all } i \in T^{\prime}\right\} .
$$

Note that $U(v, \emptyset)=U$ and that $U(v, T)=W(v, T)$ (since $W(v, T) \subseteq U$ ). By induction, it thus suffices to show that if $T^{\prime} \subseteq T$ and $T^{\prime \prime}=T^{\prime} \cup\{t\}$ with $t \in T \backslash T^{\prime}$, then the inclusion

$$
\left(\Omega_{M_{U\left(v, T^{\prime \prime}\right)}}^{\bullet}\langle\log \rangle, \nabla_{\alpha}\right) \hookrightarrow\left(\Omega_{M_{U\left(v, T^{\prime}\right)}}^{\bullet}\langle\log \rangle, \nabla_{\alpha}\right)
$$

is a quasi-isomorphism. Let $Q^{\bullet}$ be the quotient complex

$$
Q^{\bullet}=\Omega_{M_{U\left(v, T^{\prime}\right)}}^{\bullet}\langle\log \rangle / \Omega_{M_{U\left(v, T^{\prime \prime}\right)}}^{\bullet}\langle\log \rangle
$$

We show that (2.9) is a quasi-isomorphism by showing that $H^{k}\left(Q^{\bullet}\right)=0$ for all $k$.

We define a filtration $\left\{F_{p}\right\}_{p \leq v_{t}}$ on the complex $\Omega_{M_{U\left(v, T^{\prime}\right)}}\langle\log \rangle$. For $p \leq v_{t}$, let $F_{p} \Omega_{M_{U\left(v, T^{\prime}\right)}}^{k}\langle\log \rangle$ be the $\mathbb{C}[\lambda]$-submodule spanned by differential forms

$$
x^{u} \frac{d x_{i_{1}}}{x_{i_{1}}} \wedge \cdots \wedge \frac{d x_{i_{k}}}{x_{i_{k}}}
$$

satisfying $\ell_{t}(u) \geq p$. By (1.2) and (1.3), $\nabla_{\alpha}$ respects this filtration. We also denote by $F_{p}$ the induced filtrations on the subcomplex $\Omega_{M_{U\left(v, T^{\prime \prime}\right)}}\langle\log \rangle$ and the quotient complex $Q^{\bullet}$. Note that since

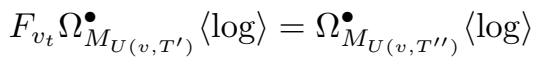

we have $F_{v_{t}} Q^{\bullet}=0$. To show that $H^{k}\left(Q^{\bullet}\right)=0$ for all $k$, it suffices to show that $H^{k}\left(\operatorname{gr}_{p} Q^{\bullet}\right)=0$ for all $k$ and $p$, where $\operatorname{gr}_{p} Q^{\bullet}$ denotes the $p$-th graded piece of the associated graded of the filtration $F_{p}$.

Write $\ell_{t}(u)=\sum_{i=1}^{n} c_{i} u_{i} \in \mathbb{Z}[u]$. Define $\rho: \Omega_{M_{U\left(v, T^{\prime}\right)}^{k}}\langle\log \rangle \rightarrow \Omega_{M_{U\left(v, T^{\prime}\right)}^{k-1}}\langle\log \rangle$ to be the $\mathbb{C}[\lambda]$-module homomorphism satisfying

$$
\rho\left(x^{u} \frac{d x_{i_{1}}}{x_{i_{1}}} \wedge \cdots \wedge \frac{d x_{i_{k}}}{x_{i_{k}}}\right)=x^{u} \sum_{j=1}^{k}(-1)^{j-1} c_{i_{j}} \frac{d x_{i_{1}}}{x_{i_{1}}} \wedge \cdots \wedge \frac{\widehat{d x_{i_{j}}}}{x_{i_{j}}} \wedge \cdots \wedge \frac{d x_{i_{k}}}{x_{i_{k}}} .
$$

It is straighforward to check that

$$
\left(\nabla_{\alpha} \circ \rho+\rho \circ \nabla_{\alpha}\right)\left(x^{u} \frac{d x_{i_{1}}}{x_{i_{1}}} \wedge \cdots \wedge \frac{d x_{i_{k}}}{x_{i_{k}}}\right)=\left(\sum_{i=1}^{n} c_{i} D_{i, \alpha}\left(x^{u}\right)\right) \frac{d x_{i_{1}}}{x_{i_{1}}} \wedge \cdots \wedge \frac{d x_{i_{k}}}{x_{i_{k}}},
$$

and a calculation using (1.1) and (1.3) shows that

$$
\sum_{i=1}^{n} c_{i} D_{i, \alpha}\left(x^{u}\right)=\ell_{t}(\alpha+u) x^{u}+\sum_{j=1}^{N} \lambda_{j} \ell_{t}\left(a^{(j)}\right) x^{u+a^{(j)}} .
$$

Suppose that the form (2.10) lies in $F_{p} \backslash F_{p+1}$. Then $\ell_{t}(\alpha+u)=\ell_{t}(\alpha)+p$, and if $\ell_{t}\left(a^{(j)}\right) \neq 0$, then $\ell_{t}\left(u+a^{(j)}\right)>p$. It follows that the second term on the right-hand side of (2.11) lies in $F_{p+1}$, so on the associated graded complex the induced map

$$
\left(\nabla_{\alpha} \circ \rho+\rho \circ \nabla_{\alpha}\right): \operatorname{gr}_{p} Q^{k} \rightarrow \operatorname{gr}_{p} Q^{k}
$$


is just multiplication by $\ell_{t}(\alpha)+p$. Since $\alpha$ is nonresonant for $A, \ell_{t}(\alpha)+p \neq 0$. Thus multiplication by a nonzero constant is homotopic to the zero map, which implies that $H^{k}\left(\operatorname{gr}_{p} Q^{\bullet}\right)=0$ for all $k$.

\section{Proof of Theorem 1.5}

In this section we assume that $f=x_{n} g\left(x_{1}, \ldots, x_{n-1}\right)$. By the remark following Theorem 1.4, we may assume that if $\alpha_{n} \in \mathbb{Z}$, then $\alpha_{n} \geq 1$. Let $R_{+}=$ $\mathbb{C}[\lambda]\left[x_{1}^{ \pm 1}, \ldots, x_{n-1}^{ \pm 1}, x_{n}\right]$. By Proposition 2.5, the inclusion

$$
\left(\Omega_{R_{+}}^{\bullet}\langle\log \rangle, \nabla_{\alpha}\right) \hookrightarrow\left(\Omega_{R^{\prime} / \mathbb{C}[\lambda]}^{\bullet}, \nabla_{\alpha}\right)
$$

is a quasi-isomorphism of complexes of $\mathcal{D}$-modules. Theorem 1.5 is then a consequence of the following result.

Proposition 3.1. If $\alpha_{n} \notin \mathbb{Z}_{\leq 0}$, then there is a quasi-isomorphism of complexes of D-modules

$$
\left(\Omega_{R_{+}}^{\bullet}\langle\log \rangle, \nabla_{\alpha}\right) \rightarrow\left(\Omega_{U / \mathbb{C}[\lambda]}^{\bullet}[-1], \widetilde{\nabla}_{\alpha}\right) .
$$

Proof. We regard $\Omega_{R_{+}}^{\bullet}\langle\log \rangle$ as the total complex associated to a certain two-row double complex: let

$$
\Omega^{k, 0}=\bigoplus_{1 \leq i_{1}<\cdots<i_{k} \leq n-1} R_{+} \frac{d x_{i_{1}}}{x_{i_{1}}} \wedge \cdots \wedge \frac{d x_{i_{k}}}{x_{i_{k}}}
$$

and let

$$
\Omega^{k, 1}=\bigoplus_{1 \leq i_{1}<\cdots<i_{k} \leq n-1} R_{+} \frac{d x_{i_{1}}}{x_{i_{1}}} \wedge \cdots \wedge \frac{d x_{i_{k}}}{x_{i_{k}}} \wedge \frac{d x_{n}}{x_{n}}
$$

Let $\partial_{h}: \Omega^{k, i} \rightarrow \Omega^{k+1, i}$ be the map

$$
\partial_{h}(\omega)=d^{\prime} \omega+\sum_{i=1}^{n-1} \alpha_{i} \frac{d x_{i}}{x_{i}} \wedge \omega+x_{n}\left(d^{\prime} g \wedge \omega\right),
$$

where $d^{\prime}$ is an exterior differentiation relative to the variables $x_{1}, \ldots, x_{n-1}$, and let $\partial_{v}: \Omega^{k, 0} \rightarrow \Omega^{k, 1}$ be the map

$$
\partial_{v}(\omega)=d^{\prime \prime} \omega+\alpha_{n} \frac{d x_{n}}{x_{n}} \wedge \omega+g d x_{n} \wedge \omega
$$

where $d^{\prime \prime}$ is an exterior differentiation relative to the variable $x_{n}$. Since $\partial_{v}$ is injective, the canonical projection $\Omega_{R_{+}}^{k}\langle\log \rangle \rightarrow \Omega^{k-1,1}$ induces a quasi-isomorphism

$$
\left(\Omega_{R_{+}}^{\bullet}\langle\log \rangle, \nabla_{\alpha}\right) \rightarrow\left(\left(\Omega^{\bullet, 1} / \partial_{v} \Omega^{\bullet, 0}\right)[-1], \partial_{h}\right)
$$

(see [13, Appendix B]).

To complete the proof of Proposition 3.1, we define a quasi-isomorphism of complexes between $\left(\Omega^{\bullet, 1} / \partial_{v} \Omega^{\bullet, 0}, \partial_{h}\right)$ and $\left(\Omega_{U / \mathbb{C}[\lambda]}^{\bullet}, \widetilde{\nabla}_{\alpha}\right)$. Define $\gamma: \Omega^{k, 1} \rightarrow \Omega_{U / \mathbb{C}[\lambda]}^{k}$ to be the $\mathbb{C}[\lambda]$-module homomorphism satisfying

$$
\gamma\left(x^{u} \frac{d x_{i_{1}}}{x_{i_{1}}} \cdots \frac{d x_{i_{k}}}{x_{i_{k}}} \frac{d x_{n}}{x_{n}}\right)=\frac{(-1)^{u_{n}}\left(\alpha_{n}\right)_{u_{n}} x_{1}^{u_{1}} \cdots x_{n-1}^{u_{n-1}}}{g^{u_{n}}} \frac{d x_{i_{1}}}{x_{i_{1}}} \cdots \frac{d x_{i_{k}}}{x_{i_{k}}},
$$

where $\left(\alpha_{n}\right)_{u_{n}}=\alpha_{n}\left(\alpha_{n}+1\right) \cdots\left(\alpha_{n}+u_{n}-1\right)$. It is straightforward to check that $\gamma$ commutes with boundary operators and hence defines a homomorphism of complexes from $\Omega^{\bullet, 1}$ to $\Omega_{U / \mathbb{C}[\lambda]}^{\bullet}$. The hypothesis that $\alpha_{n} \notin \mathbb{Z}_{\leq 0}$ implies that $\gamma$ is 
surjective, and it is straightforward to check that $\gamma\left(\partial_{v} \Omega^{k, 0}\right)=0$. It remains only to show that

$$
\operatorname{ker}\left(\gamma: \Omega^{k, 1} \rightarrow \Omega_{U / \mathbb{C}[\lambda]}^{k}\right)=\partial_{v} \Omega^{k, 0}
$$

Let $\xi \in \Omega^{k, 1}$ satisfy $\gamma(\xi)=0$. Write

$$
\xi=\sum_{i=m}^{M} x_{n}^{i} \xi_{i} \frac{d x_{n}}{x_{n}},
$$

where $\xi_{i} \in \Omega^{k, 0}$ is in the $\mathbb{C}[\lambda]$-span of the forms

$$
x_{1}^{w_{1}} \cdots x_{n-1}^{w_{n-1}} \frac{d x_{i_{1}}}{x_{i_{1}}} \wedge \cdots \wedge \frac{d x_{i_{k}}}{x_{i_{k}}}, \quad 1 \leq i_{1}<\cdots<i_{k} \leq n-1 .
$$

We prove by induction on $M-m$ that $\xi \in \partial_{v} \Omega^{k, 0}$. We have

$$
0=\gamma(\xi)=\sum_{i=m}^{M}(-1)^{i}\left(\alpha_{n}\right)_{i} \frac{\xi_{i}}{g^{i}} .
$$

Since $\left(\alpha_{n}\right)_{M} \neq 0$, this equation implies $\xi_{M}=g \eta$, where $\eta$ is in the $\mathbb{C}[\lambda]$-span of the forms (3.3). It follows that

$$
\xi=(-1)^{k} \partial_{v}\left(x_{n}^{M-1} \eta\right)-\left(\alpha_{n}+M-1\right) x_{n}^{M-1} \eta \frac{d x_{n}}{x_{n}}+\sum_{m=1}^{M-1} x_{n}^{i} \xi_{i} \frac{d x_{n}}{x_{n}} .
$$

By induction we are reduced to the case $\xi=x_{i}^{m} \xi_{m} \frac{d x_{n}}{x_{n}}$. However, in this case

$$
0=\gamma(\xi)=(-1)^{m}\left(\alpha_{n}\right)_{m} \xi_{m} / g^{m},
$$

so $\xi_{m}=0$.

If $\alpha_{n} \notin \mathbb{Z}$, there is no need to introduce the complex $\Omega_{R_{+}}^{\bullet}\langle\log \rangle$.

Proposition 3.4. If $\alpha_{n} \notin \mathbb{Z}$, then there is a quasi-isomorphism of complexes of $\mathcal{D}$-modules

$$
\left(\Omega_{R^{\prime} / \mathbb{C}[\lambda]}^{\bullet}, \nabla_{\alpha}\right) \rightarrow\left(\Omega_{U / \mathbb{C}[\lambda]}^{\bullet}[-1], \widetilde{\nabla}_{\alpha}\right)
$$

Sketch of the proof. One proceeds as in the proof of Proposition 3.1 with the complex $\Omega_{R_{+}}^{\bullet}\langle\log \rangle$ replaced by $\Omega_{R^{\prime} / \mathbb{C}[\lambda]}^{\bullet}$. One defines $\gamma$ as before with the understanding that for $u_{n}<0$

$$
\left(\alpha_{n}\right)_{u_{n}}=\left(\left(\alpha_{n}-1\right)\left(\alpha_{n}-2\right) \cdots\left(\alpha_{n}+u_{n}\right)\right)^{-1} .
$$

The proof then proceeds unchanged. (See [3, Lemma 2.5] for the details in a similar situation.)

\section{Application of N. Katz's Results}

We begin by sketching the proof that the $H^{i}\left(\Omega_{U / \mathbb{C}[\lambda]}^{\bullet}, \widetilde{\nabla}_{\alpha}\right)$ come from geometry. Let $X \subseteq \mathbb{T}_{\mathbb{C}[\lambda]}^{n}$ be the hypersurface $x_{n}^{D} g\left(x_{1}^{D}, \ldots, x_{n-1}^{D}\right)-1=0$, where $D \in \mathbb{Z}_{>0}$. Since $\Omega_{X / \mathbb{C}[\lambda]}^{1}$ is a free module with basis $\left\{d x_{i}\right\}_{i=1}^{n-1}, \mathcal{D}$ acts on global $i$-forms by acting on their coefficients relative to exterior powers of this basis. The group $\left(\boldsymbol{\mu}_{D}\right)^{n}$ acts on $X$ and its relative de Rham complex $\left(\Omega_{X / \mathbb{C}[\lambda]}^{\bullet}, d\right)$. The irreducible representations $\chi$ of $\left(\boldsymbol{\mu}_{D}\right)^{n}$ can be indexed by $n$-tuples $\left(a_{1}, \ldots, a_{n}\right), 0 \leq a_{i}<D$, so that if $\chi$ corresponds to $\left(a_{1}, \ldots, a_{n}\right)$, then there is an isomorphism of complexes of 
$\mathcal{D}$-modules $\left(\Omega_{X / \mathbb{C}[\lambda]}^{\bullet}, d\right)^{\chi} \cong\left(\Omega_{U / \mathbb{C}[\lambda]}^{\bullet}, \widetilde{\nabla}_{\alpha}\right)$, where $\alpha=\left(a_{1} / D, \ldots, a_{n} / D\right)$.) The first assertion of Corollary 1.6 then implies that

$$
\mathcal{M}_{\alpha} \cong H_{\mathrm{DR}}^{n-1}(X / \mathbb{C}[\lambda])^{\chi},
$$

which establishes the second assertion of Corollary 1.6.

Now suppose that $C=\operatorname{Spec}(A)$ is a smooth connected curve over $\mathbb{C}$ and $\phi$ : $C \rightarrow \mathbb{A}^{N}=\operatorname{Spec}(\mathbb{C}[\lambda])$ is a morphism. Let $X^{\prime}$ be the pullback of $X$ to a variety over $C$, i.e., $X^{\prime}=A \otimes_{\mathbb{C}[\lambda]} X$. Then $X^{\prime}$ is the hypersurface in $\mathbb{T}_{A}^{n}$ defined by the equation $x_{n}^{D} g^{\phi}\left(x_{1}^{D}, \ldots, x_{n-1}^{D}\right)-1=0$, where $g^{\phi} \in A\left[x_{1}^{ \pm 1}, \ldots, x_{n-1}^{ \pm 1}\right]$ is the Laurent polynomial obtained from $g$ by applying $\phi$ to its coefficients (by abuse of notation, we also denote by $\phi$ the homomorphism $\mathbb{C}[\lambda] \rightarrow A$ corresponding to $\phi: C \rightarrow \mathbb{A}^{N}$ ). The varieties $X$ and $X^{\prime}$ are smooth affine schemes whose de Rham cohomology can be computed as the cohomology of the complex of global sections of the de Rham complex. By the right-exactness of tensor products, one has

$$
\phi^{*}\left(H_{\mathrm{DR}}^{n-1}(X / \mathbb{C}[\lambda])\right) \cong H_{\mathrm{DR}}^{n-1}\left(X^{\prime} / A\right)
$$

It follows from [9, Section 14] that $H_{\mathrm{DR}}^{n-1}\left(X^{\prime} / A\right)$ has regular singular points and quasi-unipotent local monodromy at infinity (i.e., at all points of the quotient field of $A$ ). Therefore $H_{\mathrm{DR}}^{n-1}(X / \mathbb{C}[\lambda])$ has regular singular points and quasi-unipotent local monodromy at infinity (in the sense of [11, Section VIII]). Equation (4.1) then implies that $\mathcal{M}_{\alpha}$ has regular singular points and quasi-unipotent local monodromy at infinity.

To apply the results of [10], we observe that the results of this paper are valid when one replaces $\mathbb{C}[\lambda]$ by $\mathbb{C}(\lambda)$. Let $\overline{\mathcal{D}}$ denote the ring of differential operators with coefficients in $\mathbb{C}(\lambda)$ and define

$$
\overline{\mathcal{M}}_{\alpha}=\overline{\mathcal{D}} /\left(\sum_{l \in L} \overline{\mathcal{D}} \square_{l}+\sum_{i=1}^{n} \overline{\mathcal{D}} Z_{i, \alpha}\right) .
$$

Put

$$
\bar{R}=\mathbb{C}(\lambda)\left[x_{1}^{ \pm 1}, \ldots, x_{n}^{ \pm 1}\right]
$$

the coordinate ring of the $n$-torus $\mathbb{T}_{\mathbb{C}(\lambda)}$. The proof of Theorem 1.4 establishes the following result.

Proposition 4.3. If $\alpha$ is nonresonant for $A$, then $\bar{M}_{\alpha} \cong H^{n}\left(\Omega_{\bar{R} / \mathbb{C}(\lambda)}, \nabla_{\alpha}\right)$ as $\overline{\mathcal{D}}$-modules.

In the situation of Theorem 1.5, let $\bar{U} \subseteq \mathbb{T}_{\mathbb{C}(\lambda)}^{n}$ be the open set where $g$ is nonvanishing. Then we have the following result.

Proposition 4.4. Suppose $f=x_{n} g\left(x_{1}, \ldots, x_{n-1}\right)$ and $\alpha$ is nonresonant for $A$. For all $i$ there are $\overline{\mathcal{D}}$-module isomorphisms

$$
H^{i}\left(\Omega_{\bar{R} / \mathbb{C}(\lambda)}^{\bullet}, \nabla_{\alpha}\right) \cong H^{i-1}\left(\Omega_{\bar{U} / \mathbb{C}(\lambda)}^{\bullet}, \tilde{\nabla}_{\alpha}\right) .
$$

Combining these propositions gives the following result.

Corollary 4.5. Suppose $f=x_{n} g\left(x_{1}, \ldots, x_{n-1}\right)$ and $\alpha$ is nonresonant for $A$. There is an isomorphism of $\overline{\mathcal{D}}$-modules $\overline{\mathcal{M}}_{\alpha} \cong H^{n-1}\left(\Omega_{\bar{U} / \mathbb{C}(\lambda)}^{\bullet}, \tilde{\nabla}_{\alpha}\right)$. If in addition $\alpha \in \mathbb{Q}^{n}$, then $\overline{\mathcal{M}}_{\alpha}$ comes from geometry. 
Explicitly, letting $\bar{X} \subseteq \mathbb{T}_{\mathbb{C}(\lambda)}^{n}$ be the hypersurface $x_{n}^{D} g\left(x_{1}^{D}, \ldots, x_{n-1}^{D}\right)-1=0$, we have (corresponding to equation (4.1))

$$
\overline{\mathcal{M}}_{\alpha} \cong H_{\mathrm{DR}}^{n-1}(\bar{X} / \mathbb{C}(\lambda))^{\chi}
$$

By [10, Theorem 5.7] $H_{\mathrm{DR}}^{n-1}(\bar{X} / \mathbb{C}(\lambda))^{\chi}$ has a full set of polynomial solutions modulo $p$ for almost all primes $p$ if and only if it has a full set of algebraic solutions. Note that the solution sets of $\mathcal{M}_{\alpha}$ and $\overline{\mathcal{M}}_{\alpha}$ in the algebraic closure of $\mathbb{C}(\lambda)$ are identical. From equation (4.6), we then get the following result.

Corollary 4.7. Suppose $f=x_{n} g\left(x_{1}, \ldots, x_{n-1}\right)$ and $\alpha \in \mathbb{Q}^{n}$ is nonresonant for $A$. The hypergeometric $\mathcal{D}$-module $\mathcal{M}_{\alpha}$ has a full set of polynomial solutions modulo $p$ for almost all primes $p$ if and only if it has a full set of algebraic solutions.

\section{REFERENCES}

[1] Adolphson, Alan. Hypergeometric functions and rings generated by monomials. Duke Math. J. 73 (1994), no. 2, 269-290. MR.1262208 (96c:33020)

[2] Adolphson, Alan. Higher solutions of hypergeometric systems and Dwork cohomology. Rend. Sem. Mat. Univ. Padova 101 (1999), 179-190. MR1705287 (2001b:14032)

[3] Adolphson, Alan; Sperber, Steven. On twisted de Rham cohomology. Nagoya Math. J. 146 (1997), 55-81. MR1460954 (98k:14027)

[4] Beukers, Frits. Algebraic A-hypergeometric functions. Invent. Math. 180 (2010), no. 3, 589610. MR2609251

[5] Dwork, Bernard. Generalized hypergeometric functions. Oxford Mathematical Monographs. Oxford Science Publications. The Clarendon Press, Oxford University Press, New York, 1990. MR.1085482(92h:14006)

[6] Dwork, B.; Loeser, F. Hypergeometric series. Japan. J. Math. (N.S.) 19 (1993), no. 1, 81-129. MR.1231511 (95f:33013)

[7] Katz, Nicholas. Thesis (1966), Princeton University.

[8] Katz, Nicholas. On the differential equations satisfied by period matrices. Inst. Hautes Études Sci. Publ. Math. No. 35 (1968), 223-258. MR0242841 (39:4168)

[9] Katz, Nicholas. Nilpotent connections and the monodromy theorem: Applications of a result of Turrittin. Inst. Hautes Études Sci. Publ. Math. No. 39 (1970), 175-232. MR0291177 $(45: 271)$

[10] Katz, Nicholas. Algebraic solutions of differential equations ( $p$-curvature and the Hodge filtration). Invent. Math. 18 (1972), 1-118. MR0337959 (49:2728)

[11] Katz, Nicholas. A conjecture in the arithmetic theory of differential equations. Bull. Soc. Math. France 110 (1982), no. 2, 203-239. MR667751 (84h:14014a)

[12] Katz, Nicholas M. Corrections to: "A conjecture in the arithmetic theory of differential equations". Bull. Soc. Math. France 110 (1982), no. 3, 347-348. MR688039 (84h:14014b)

[13] Matsumura, Hideyuki. Commutative ring theory. Translated from the Japanese by M. Reid. Cambridge Studies in Advanced Mathematics, 8. Cambridge University Press, Cambridge, 1986. MR879273 (88h:13001)

[14] Schulze, Mathias; Walther, Uli. Hypergeometric D-modules and twisted Gauss-Manin systems. J. Algebra 322 (2009), no. 9, 3392-3409. MR2567427 (2010m:14028)

Department of Mathematics, Oklahoma State University, Stillwater, Oklahoma 74078

E-mail address: adolphs@math.okstate.edu

School of Mathematics, University of Minnesota, Minneapolis, Minnesota 55455

E-mail address: sperber@math.umn.edu 